\title{
The Avatar and The Player: Understanding the Relationship Beyond the Screen
}

\author{
Alison Gazzard \\ University of Hertfordshire \\ College Lane, Hatfield, UK \\ A.J.Gazzard@herts.ac.uk
}

\begin{abstract}
This paper looks at the concept of the avatar in terms of our interaction and viewpoints. It proposes a vocabulary of terms which analytically describe the player:avatar relationship as opposed to the player:screen relationship, emphasising the performative rather than the representational elements. In place of transposing cinematographic concepts such as 'first person' or 'third person' to the discussion of avatars, as has been the case in the past, the paper proposes the idea of the 'altered positions' of the avatar, whether that be playing 'within' the character' or 'outside' of the character. These concepts link to the avatarial display and avatarial presence of a game character and emphasise how the various 'positions' effect our movements and experience within the game world, and help us understand how we, as players, are 'performing' through our avatars, engaging in and enjoying a player->controller->avatar experience that is essentially aesthetic and emotional.
\end{abstract}

\section{INTRODUCTION}

The body will always be an issue in discussing virtual worlds. We can't ignore our physical presence completely even when 'immersed' in the virtual world. It is 'I as an embodied self' that moves the controller and presses the button to 'walk' and 'jump' through various game levels. It is 'I as an embodied self' that breathes in and out whilst venturing through Osmose. It is 'I as an embodied self' that moves the mouse to double click to open the folder on screen. Although we are controlling these actions, it is a representation on the screen that is acknowledging our input and showing us the actions are being performed.

Separating and discussing the avatar in terms of presence and display, shows how we as users/players perform through/with/as the avatar. By recognising the avatar as more than the 'character' or 'protagonist' so often referenced in discussing virtual worlds and digital media, we can start exploring how avatars exist beyond their displayed representations on screen, how the exist as performed entities. Seeing how, on entering the 'magic circle' [1] of a game world, player's/user's 'performed out' actions can translate to the actions/agency of the avatar.

The word avatar has been used in digital games since Habitat was created in the 1980s. It was the first online world to depict the player's character as an onscreen graphic, referred to by Morningstar and Farmer as an 'avatar'.
'Avatars can move around, pick up, put down and manipulate objects, talk to each other, and gesture, each under the control of the individual player' [2]

The word avatar is now used commonly in describing characters in $2 \mathrm{D}$ and $3 \mathrm{D}$ virtual worlds but is rarely discussed in terms of anything other than its representations. What happens when there is no character represented in our virtual world? Does the notion of the avatar disappear and if so, how do we understand what we are controlling in its absence?

Representations of the user/player in many serious games are now increasingly changeable, with onscreen cursors turning into seemingly disembodied arms depending on the situation, such as in TruSim's Interactive Trauma Trainer, where the user's avatar changes from the act of selecting with a cursor to the act of administering treatment through a displayed arm. Although the emphasis is still on the player to make decisions and act on them during the simulated experience, their own representation changes, creating a discussion about whether the player's avatar is still present in both depicted scenarios. The cursor shifts to the previously termed ' $1{ }^{\text {st }}$ Person' position, from that of a cursor display and it is these terms of ' $1{ }^{\text {st }}$ Person' and ' $3{ }^{\text {rd }}$ Person' that have been remediated from film and literary theory that will be a focus of this discussion by shifting the emphasis to the avatarial display and presence of our virtual selves.

In order to discuss character:representation, avatar:experience we need to first examine what is an avatar, and how can we discuss a players performance through a character whilst moving through/with/as a variety of positions as that avatar?

\section{WHAT IS AN AVATAR?}

The word 'avatar' has been used long before digital technology and virtual worlds. It is rooted in religion, particularly Hinduism, where Hindu's have believed that the god, Vishnu, materialises in a range of avatars. Avatars can be defined as; 'the appearance of a god in human or animal form' [3]

These definitions are not the same as the 'avatar' we refer to within the digital realm, but they can be used to start to understand how the word can be used in with the vocabulary of digital media. The main difference between the 'real- 
world' avatar as opposed to the one we now refer to in digital media, is that we, as users/players can not be wholly embodied into/onto what is occurring onscreen. The displayed character has to be manipulated through a controller, mouse or other external input device by the player so that it can be moved and repositioned. In the story of Leda and the swan in Greek mythology, the god Zeus becomes the swan. His whole body is replaced by that of the swan, and his actions are translated into those of the new creature he embodies for that period of time. His positioning became the swan's positioning; he was not present separately as both himself and as a swan in two separate places. This is not possible in a virtual world. The screen does not allow for the translation of our bodies into the virtual body, therefore we are present in dual realities, of both the quotidian and the virtual world. Our bodies can, however, be extended through the use of input devices to allow us to perform through our virtual avatars.

This is the new relationship between player and avatar in the digital age. The association with embodiment and being able to exist in another form, sees the element of projecting the beliefs of one being onto something else and using the word 'avatar' in the 'digital'. Although its original meaning has been slightly altered, many elements remain true. Here an avatar is an 'owned' or 'inhabited' character in the space which we 'perform through, with or as'. We choose our avatars, or they are created for us. We choose to play as them, to move as them and our actions are translated on to them.

'The avatar is the embodied manifestation of the player's engagement with the gameworld; it is the player incarnated' [4]

It is through using an avatar that feedback loops of interaction can be seen and experienced. A sense of 'agency' [5] is evoked and the responsibility of the players actions towards what is happening starts to be generated. The avatar is an important concept in our learning and development through serious games. Understanding the onscreen depiction of the user/player and the feedback of the player->controller->avatar movement shows the user/player their role within the virtual environment. It is through this recognising this feedback loop that users/players can develop their understanding as to what they are manipulating in the virtual world and why. The displayed avatar may be seen as a common element of some serious games but players use the avatar to gain rewards and/or learn about the virtual space they are now in, therefore users/players have an attachment to them, an investment in them. This attachment is key to how we understand avatars and what separates them from the representational elements displayed to us onscreen. To be able to understand how the avatar exists as more than displayed representation, and as part of performance and emotional attachment, I will briefly discuss the avatar outside of the digital realm.

Game worlds/spaces exist outside of the virtual. Playing games of Dungeons and Dragons, Monopoly, chess or playing with Barbie or Action Man, all allow us to enter a game scenario. Through crossing over into the magic circle, the pieces on the board of Ludo can become our avatar, as can Barbie. Yet at the same time, playing with dolls can also be seen as non-avatarial. When playing with Barbie or Action Man, we are not always becoming them. We are controlling them but can recognise them as something outside of us. They are more like 'friends' than an identification of who we are. We care for them but not necessarily about them and this can be seen through the act of "Projective Play".

'Projective Play happens when the child discovers the world outside themselves through toys, dolls and other play objects. The child uses these objects to represent other objects so a doll becomes a baby and a stick becomes a sword' [6]

So instead of the objects being incarnations of the player/child, as an avatar would be, the objects take on different meanings for the child to work out the world around them. This is re-iterated in playing as the Top Hat in Monopoly. The Top Hat is non-avatarial, as by itself it cannot move around the board. The piece is stationary until the dice is thrown, which in turn affects the decisions in the game world and how far and where the player can move the piece on the board. The Top Hat can be seen as a character or display, and the dice as a positioning/moving device so jointly they have some traits of an avatar but separately they have different uses and means. This leads us consider, what are the characteristics of an avatar?

\section{CHARACTERISTICS OF AVATARS}

As discussed previously, the avatar is more than what is shown within the gameworld. As well as seeing our character, we are able to move it, make it respond to events in the game space, and we invest in it. It is these components that construct the avatar, and can be discussed as these four characteristics:

1. Locus - it's place in its world and how that is communicated to the user/player.

2. Agency - the ability to effect an action in its world.

3. Empathy - how much the player/user relates to and/or cares about what they are affecting in the world.

4. Player Character - who am I within the world? What can I do? What do I represent? ${ }^{1}$

Avatars consist of parts of these traits. Not all avatars will have the same levels of each characteristic but to be an avatar, they will be constructed of all of these qualities. The main components of the avatar are having a sense of agency and seeing the result of those actions. This also encompasses

\footnotetext{
${ }^{1}$ Chaim Gingold discusses characteristics similar to these in writing about "The structure of point of view in participatory media..." (Gingold 2003) His variations focus on what we can see through the screen and how this works with the avatar. The focus is not so much on how we are controlling a character, but as to how we see what we are controlling.
} 
changing the location of the avatar and how much we care about what we are affecting. Seeing the results of our actions can be described by using the term 'avatarial presence'. This differs to the representation of avatar that can be termed 'avatarial display'. By using and separating these two terms we can start to see how the experience of the avatar can be described and discussed in different positionings of the avatar, as offered by different genre of videogames and virtual spaces. There will always be an 'avatarial' presence whilst experiencing interactive immersive environments, this is the feedback loop described earlier on that allows us to see the resulting actions of changing the signals of the input device. Viewing this 'presence' separately, we can start to understand how we can have 'avatarial presence' without the need for an iconic representation of character through 'avatarial display'. We need a display to inform us of our actions, and to re-iterate our 'presence' within the system. These two elements combined feedback multiple scenarios in serious games to help us learn and progress. The avatar in many ways can act as our 'status bar' as to our progress, giving the player feedback on right or wrong actions, or simply which buttons/keys trigger certain events within the virtual space.

\section{ALTERED POSITIONS}

In changing how the 'point of view' of the user/player is discussed, we are able to discuss the experience of how we experience the avatar in the virtual world, rather than the screen we are seeing them through. Therefore instead of 'points of view', we can discuss these as 'altered positions' of the avatar. These positions start to describe the player:avatar relationship as opposed to the player:screen relationship and reinforce the performative aspects of the avatar in these various positions rather than the representational elements. They also allow for discussions about 'avatarial presence' and 'avatarial display' in various virtual world scenarios, and how each of these may differ in viewing and experiencing the avatar in these ways. In starting to develop a vocabulary of avatars in this way, I list four 'altered positions' as outlined:

$1^{\text {st }}$ Altered Position - no or limited avatarial display e.g. on screen cursor only $2^{\text {nd }}$ Altered Position - partial avatarial display e.g. arm, equipment, hand, etc $3^{\text {rd }}$ Altered Position - full avatarial display. View of avatar is linked to camera movement.

$4^{\text {th }}$ Altered Position - full avatarial display.

Player/user has an omniscopic view of the virtual world. Avatar is not linked to camera movement. Avatars can exist singularly or in teams.

The following diagrams show, where the player/user 'sits' in terms of the experience when immersed in one of the various states. They consist of the 'social world', which is the quotidian lives we exist within (the real world we are experiencing the virtual world in), the 'magic circle' [1] as defined by Huizinga (the space where we enter the game world) and the 'story', the videogame/artefact itself.

The diagrams below show what is happening in each Altered Position in terms of the avatarial display and a depicted representation (as represented by the grey ellipses):
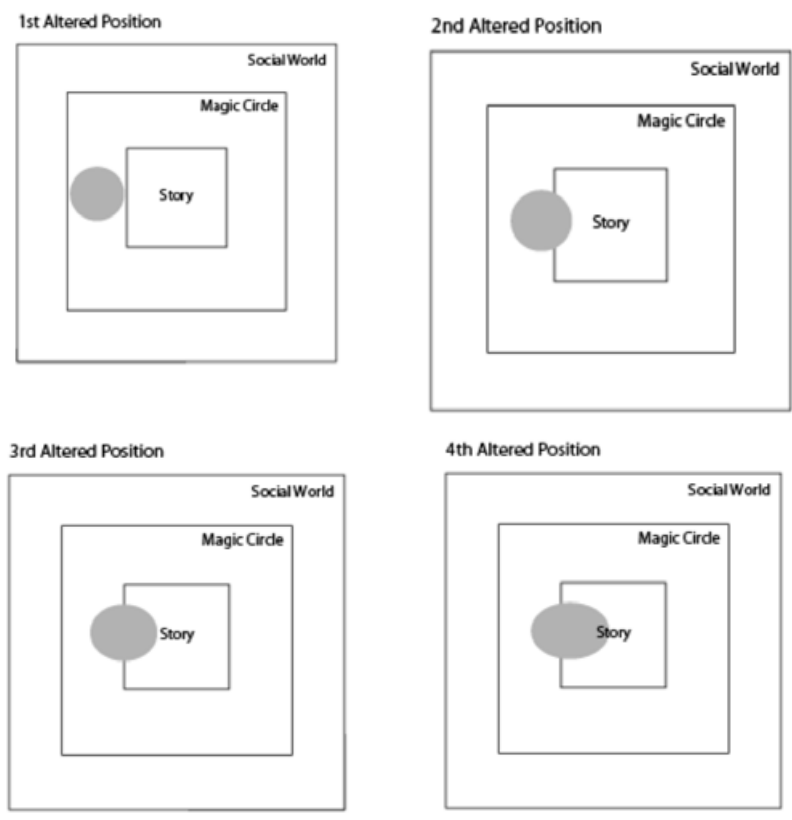

Fig 1. Altered Positions

- In the $1^{\text {st }}$ Altered Position the user/player only has display in the magic circle. This shows that they have entered the game world and can start to affect it, but they are not displayed within the actual story space. The user/player may be displayed in the story space in terms of a cursor such as when selecting objects to help patients in TruSim's Triage Trainer.

- In the $2^{\text {nd }}$ Altered Position the user/player's display is still shown in the magic circle, but it now starting to become visible in the story world. This is through a slight avatarial representation such as the showing of a characters fingers/hand/arm when administering procedures in TruSim's Interactive Trauma Trainer or picking up and placing objects.

- In the $3^{\text {rd }}$ Altered Position the avatarial display is greater in the story world than in the $2^{\text {nd }}$ position due to the user/player seeing more of the avatar. The use/player will be able to view most of the avatar yet will be linked to it through having a linked avatarial display. The view of the view will be seen through guiding the avatar on screen such as in SecondLife.

- In the $4^{\text {th }}$ Altered Position, the display of the avatar is slightly greater than in the $3^{\text {rd }}$ position. This is due 
to the user/player being able to view the displayed avatar and it's setting in more detail, as they are 'dualled' rather than linked. Having a dual relationship also means other avatars can be viewed such as in a team situation where the user/player is controlling a team rather than a single avatar.

Due to the 'magic circle' existing as part of the 'social world', the onscreen avatar is linked through the performance of the player in the real world. The real world actions of the controller feedback into the games system show us how we are acting within the game space, and it is only the 'display' of the avatar that changes in each of these positions. Our ability to effect the game world remains much the same, it is the signs of what we are affecting and how we can affect it, that change in each of the scenarios. The user interface for each scenario differs slightly to work with the 'avatarial display' to provide us with our health status, point scores, amount of lives left, and maps showing us our positioning in the world. These are objects that change depending on the game scenario, but work with the 'avatarial display' to give us our virtual presence within the game space.

\section{CONCLUSION}

This paper attempts to open up ways of discussing the avatar in relation to various games, separating previous vocabularies associated with other media. It is particularly important due to the number of serious games using cursor control instead of depicted characters that the avatar is recognised as more than an onscreen representation, especially as "Serious games and virtual worlds offer great potential for learner to step inside the screen of their imagining..."[7]. Understanding the avatar as a combination of both presence and display (even in a limited form) shows how users/players are still able to relate to and empathise with onscreen scenarios to aid their learning and development. In doing so, it is now possible to understand how various games and simulations do not have traditional 'fixed' avatarial representations and the display of the avatar can be altered during play, or may not fall into a definitive category in the first place. Each Altered Position can now exist on a scale, allowing artefacts to be positioned anywhere from the $1^{\text {st }}$ up to the $4^{\text {th }}$ Position depending on their avatarial display and associated presence.

The Nintendo Wii highlights how games may not have fixed ' $1{ }^{\text {st }}$ person' or ' 3 rd person' displays, therefore by using 'altered positions' it is easier to discuss the avatarial display on a sliding scale. Games such as the boxing game within Wii Sports, create an issue in how the altered position would be discussed. In the boxing game, the character is viewed from the $3^{\text {rd }}$ Altered Position, yet at the same time, the displayed character is only roughly $30 \%$ of its original opacity.

This causes the character to appear as a 'ghost-like' image of its original character. In this instance it is part way to being in the $2^{\text {nd }}$ Altered Position as the displayed character is used to show how you are punching your opponent. It could be said that this game sits somewhere between the $2^{\text {nd }}$ and $3^{\text {rd }}$ Positions on a sliding scale. The character is needed in the $3^{\text {rd }}$ Altered Position to act as a status bar allowing the player to see that they have been hit by their opponent, through the displayed action of the avatar onscreen, yet in order to perform through the avatar, the transparency is needed in to see their opponent in the game and understand how to defeat the other character.

This transparency of the onscreen character can also be seen in the jogging game in Wii Fit, where again, the avatar is displayed at a certain transparency, yet the character can still be seen on screen. Through the displayed avatar 'jogging' and being displayed with a similar motion to that of the player, there is a clear relationship between both player and avatar. The avatar is both in the $2^{\text {nd }}$ and $3^{\text {rd }}$ Altered Positions, allowing the player to relate to the onscreen avatar, and see the road ahead that they are running along in the virtual world.

With the growth in new game/play technologies constantly evolving, players can now start to understand ways of playing games. Instead of relying on filmic conventions and points of view, we can start to understand our relationships to our avatars and how we can use them to play within the game space. Removal of the displayed character does not remove the avatar.

One pleasure of using interactive content is the sense of agency in seeing how what you are doing effects the environment you are moving through. This can happen through having an 'avatarial presence', and allowing the user/player to play without having to project their actions onto displayed character on the screen. By understanding the avatar as having these two separate distinctions of 'presence' and 'display', we can recognise how virtual spaces can be created and explored, as well as understanding the various motivations and experiences of players in doing so.

\section{REFERENCES}

[1] J. Huizinga, Homoludens: A Study of the Play Element in Culture, New York: J \& J Harper Editions, 1970

[2] C. Morningstar and F.R. Farmer, "The Lessons of Lucasfilm's Habitat", in The First International Conference on Cyberspace, MIT Press: University of Texas, 1990

[3] Collins English Dictionary. [cited 2007 1st October]; Available from: http://www.collinslanguage.com/. 2007

[4] R. Klevje, "What is the avatar?", in Information Science and Media Studies, University of Bergen: Bergen. 2006

[5] J. Murray, Hamlet on the Holodeck, New York: The Free Press. 1997

[6] A. Cattanach, Introduction to Play Therapy, New York: Routledge, 2003

[7] S. de Freitas, Emerging Trends in Serious Games and Virtual Worlds. Becta: Emerging Technologies for Learning, 2008. 